\title{
Elise Dennis wins ABC Best Paper Award
}

\author{
Nicola Oberbeckmann-Winter ${ }^{1}$ (I)
}

Received: 21 May 2015 / Accepted: 26 May 2015 /Published online: 25 June 2015

(C) Springer-Verlag Berlin Heidelberg 2015

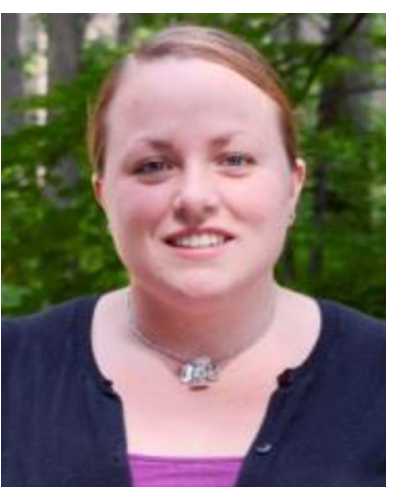

Elise Dennis, born 1988, is currently a PhD candidate in analytical chemistry at Indiana University under the direction of Professor Gary M. Hieftje. She has authored/co-authored eight peer-reviewed publications, is an inventor on two patents, and has presented two invited lectures and eight additional talks/posters at various scientific conferences. Prior to graduate school, Elise received her BS with honors from the College of William and Mary, where she performed research in the laboratory of Professor John C. Poutsma, and presented a poster on her research at a regional meeting of the American Chemical Society. In addition to completion of an honors BS thesis, Elise has received several recognitions for her academic accomplishments, including the 2014 Barbara Stull Graduate Student Award from the Society of Applied Spectroscopy, a Robert and Marjorie Mann Chair Fellowship, an REU summer research fellowship sponsored by NSF, a Howard Hughes Medical Institute Freshman Research Fellowship, a Metacyt Fellowship, an Indiana University Women in Chemistry Travel Award, and an Indiana University Provost Travel Award. Apart from her research duties, Elise also serves as the current treasurer of the Indiana Section of the Society of Applied Spectroscopy.

Nicola Oberbeckmann-Winter

abc@springer.com

1 Analytical and Bioanalytical Chemistry, Springer, Tiergartenstrasse 17, 69121 Heidelberg, Germany
The ABC Best Paper Award 2014 for outstanding work published in Analytical and Bioanalytical Chemistry (ABC) goes to Elise Dennis (27), who is lead author of the paper "ZoomTOFMS: addition of a constant-momentum-acceleration 'zoom' mode to time-of-flight mass spectrometry", which presents the performance of a new concept called zoom-time-offlight mass spectrometry (zoom-TOFMS). Her study demonstrates the unique advantages of alternating between constantmomentum acceleration and conventional, constant-energy acceleration, which include an improvement in mass resolution without a loss in instrument sensitivity. The new method is shown to be amenable to retrofitting onto existing time-offlight mass spectrometers.

Accompanied by 1500 euros, the award is sponsored by Springer to honor exceptional young scientists and to stimulate their research careers. The article "Zoom-TOFMS: addition of a constant-momentum-acceleration 'zoom' mode to time-of-flight mass spectrometry" by Elise A. Dennis, Alexander W. Gundlach-Graham, Steven J. Ray, Christie G. Enke, Charles J. Barinaga, David W. Koppenaal, and Gary M. Hieftje is available online at http://link.springer.com/article/ $10.1007 / \mathrm{s} 00216-014-7875-8$ or can be requested as a pdf.

\section{Who is Elise Dennis?}

Elise Dennis talks about her research, her motivations, and about herself to Nicola Oberbeckmann-Winter.

\section{What was your motivation to do research on zoom-time-of-flight mass spectrometry?}

Increased mass resolution is often necessary to solve analytical problems addressed with mass spectrometric techniques, 
but it almost always comes at a cost (i.e., lower throughput or repetition rate). I was motivated to develop zoom-time-of-flight mass spectrometry (zoomTOFMS) as a low-cost alternative to commercially available high-resolution TOFMS systems that also helps overcome the tradeoff between mass resolution and throughput. I see zoom-TOFMS as a relatively simple, easily accessible, value-added function that can be added to existing instrumentation.

\section{Why do you think your work merits the $\mathrm{ABC}$ Best Paper Award?}

The results presented in the paper outline the operation and initial results from zoom-TOFMS, a new type of mass spectrometry that has not been previously described. Our results show that a commercially available TOFMS instrument can be retrofitted for zoom-TOFMS operation to provide both increased mass resolution and instrumental duty factor for a defined mass-spectral range.

\section{How does the Award-winning work relate to your PhD research?}

My current $\mathrm{PhD}$ research is focused on the development of two novel velocity-based mass spectrometry systems: zoom-TOFMS and distance-of-flight mass spectrometry (DOFMS). As the award-winning article is the flagship paper on zoom-TOFMS, the results presented are very closely related to my overall dissertation research.

\section{How would you explain your research to your child?}

If I had a child, I would explain that everything we see around us is made up of small building blocks called molecules and atoms. In my research, I am developing a new way to find out what those building blocks are so that we can better understand the world around us.

\section{What's the trickiest problem you've had to overcome in your research? How did you solve it?}

Problems arose during the course of experiments that were not rooted in chemistry, but in complex electronic systems that allow the zoom-TOFMS instrument to function. I would say that learning to troubleshoot these systems effectively and efficiently to find the cause of a problem was the most challenging part of my research.

\section{Where do you see your field headed and how do you see it influencing bioanalytical research?}

Although instrumentation development is now largely done by instrument companies, I think that continued work in academic and independent labs is important because the motivation is often curiosity. In the case of zoom-TOFMS, we developed the technique as an offshoot of DOFMS to see if constant-momentum acceleration with energy focusing could be applied to benefit TOFMS systems. At least for me, the early part of the instrument development was just to see if the idea would work. Now, I can see zoom-TOFMS applied to increase the temporal resolution of chromatographic separations or to improve the quantification of metal centers in metalloproteins with increased throughput.

Which incident/discovery has proved most valuable for your own research? Which incident/discovery most inspired you during your education and scientific career? Which recent discovery might prove most valuable to the field of (bio-)analytical research or beyond?

- The basis of zoom-TOFMS is energy-focusing constant-momentum acceleration (CMA), an idea that was first explored by Chris Enke and Gareth Dobson in 2007 (Anal. Chem. (2007) 79:86508661). Without Enke and Dobson's reconsideration of CMA, which was originally employed for TOFMS much earlier and dismissed (Rev. Sci. Instrum. (1953) 24(8):616-617), neither DOFMS nor zoom-TOFMS would be possible.

- The development that comes to mind is the invention of the inductively coupled plasma (ICP). The ability to create and sustain a plasma in the laboratory that is roughly the temperature of the sun in order to study the composition of samples has really changed how analytical chemists approach the analysis of metals.

- I think the development of mass cytometry with metalconjugated antibodies as an alternative to conventional 
flow cytometry will prove invaluable to bioanalytical research in the coming years. The ability to run assays with tens of antibodies to study proteins and biomarkers allows more information to be gathered per experiment; it will be exciting to see how the technology develops.

\section{What was the best/worst advice you ever received?}

The best advice I ever received was from my undergraduate advisor (Professor John Poutsma) who encouraged me to go to graduate school.

\section{Whom do you admire the most or who inspired you the most and why?}

I would have to say my $\mathrm{PhD}$ advisor, Professor Gary Hieftje, and the senior research scientist in the Hieftje group, Dr.
Steven Ray. They have both been incredibly supportive throughout my time at Indiana University and provided a wealth of knowledge and ideas that make for a wonderful research environment.

\section{What are your future plans?}

I am currently in the process of finishing up my dissertation and looking for a position after graduation. I would like to continue to be actively involved in atomic mass spectrometry research.

\section{What do you do in your spare time?}

In my spare time I enjoy hiking, camping, and spending time with friends. 